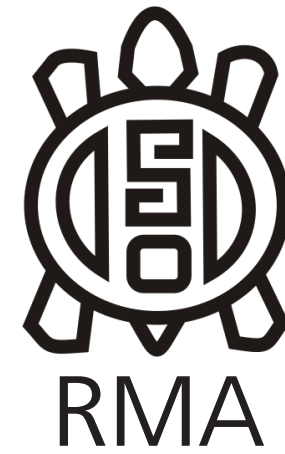

Dossier

\title{
Experimentos y esquemas diacríticos para explorar técnicas de talla unifacial del Holoceno temprano en el noroeste de Santa Cruz
}

\author{
Experiments and diacritical schemes to explore unifacial flaking \\ techniques of the early Holocene in northwest Santa Cruz
}

María T. Civalero* y Hugo G. Nami**

*CONICET-Instituto Nacional de Antropología y Pensamiento Latinoamericano-Universidad de Buenos Aires. Email: mtcivalero@gmail.com

** CONICET-Instituto de Geociencias Básicas, Aplicadas y Ambientales de Buenos Aires, Laboratorio de Geofísica "Daniel A. Valencio", Departamento de Ciencias Geológicas, Facultad de Ciencias Exactas y Naturales, Universidad de Buenos Aires. Email: hgnami@fulbrightmail.org

\begin{abstract}
Resumen
En el registro arqueológico del Holoceno temprano del noroeste de la provincia de Santa Cruz se identificó una modalidad peculiar de manufactura de instrumentos líticos unifaciales. En su secuencia de reducción las formas-bases se preformaron con lascados unifaciales-parcial o totalmente extendidos- y se las finalizaba con retoques escamosos cuya profundidad normalmente no excede los $\sim 10 \mathrm{~mm}$. Con el propósito de profundizar en el conocimiento de su manufactura se efectuaron experimentos replicativos y análisis diacríticos de los especímenes experimentales y arqueológicos. Los resultados obtenidos muestran que los lascados total o parcialmente extendidos corresponden a una acción preconcebida y deliberada tendiente a la formatización del soporte y no son accidentales ni resultan de las reactivaciones. Así, se refuerza la posición de que esta manera de confeccionar utensilios correspondería a un estilo técnico compartido por las ocupaciones del Holoceno temprano en la Patagonia centro-meridional.
\end{abstract}

Palabras clave: Tecnología lítica experimental; Análisis diacrítico; Secuencia de reducción; Instrumentos unifaciales; Patagonia centro-meridional.

\begin{abstract}
In the early Holocene archaeological record in Northwest Santa Cruz province, a peculiar modality of manufacture of unifacial lithic artefacts was identified. In its reduction sequence, the flake blanks were flaked by detaching totally or partially covering flakes and then finished by scalar retouches of about $10 \mathrm{~mm}$. In order to deepen in the knowledge of its manufacture, replicative experiments, and diacritic analysis were performed on the experimental and archaeological specimens. The obtained results show that the totally or partially covering flake-scars correspond to a preconceived and deliberate action tending to shape the flake blank and are not accidental or result from the resharpening. In this way, the research reinforced that this way of making artefacts may correspond to a technical style shared by the early Holocene occupations in central-southern Patagonia.
\end{abstract}

Keywords: Experimental lithic technology; Diacritic analysis; Reduction sequence; Unifacial artefacts; Central-southern Patagonia.

En el registro arqueológico del Holoceno temprano del noroeste de la provincia de Santa Cruz, recientemente se identificó una modalidad peculiar de manufactura de instrumentos líticos unifaciales. En su secuencia de reducción los soportes o formas-base se preformaron con lascados unifaciales -parcial o totalmente extendidos- y se las finalizaba con retoques escamosos cuya profundidad normalmente no excede los $\sim 10 \mathrm{~mm}$. Los artefactos fueron observados en sitios del área lacustre cordillerana, en los contrafuertes de la Meseta Central de Santa Cruz y otros lugares de la Patagonia centro-meridional (Civalero 2016,
Nami y Civalero 2016, 2017, Civalero y Nami 2018, Hermo et al. 2018).

El propósito de esta investigación fue profundizar en el conocimiento de la cadena de producción de los materiales utilizando dos metodologías complementarias. Por un lado, desde el campo disciplinar de la tecnología lítica experimental se llevaron a cabo experimentos replicativos (Nami 2010, 2011, 2018); por otro, se efectuó el análisis diacrítico de los especímenes experimentales y arqueológicos con el objetivo de reconocer las series 
de extracciones de lascas; de esta manera, comprender los rasgos de su superposición para conocer más acabadamente su secuencia de confección (Baena Preysler y Cuartero 2006). Fruto de esta pesquisa, este artículo brinda los resultados obtenidos utilizando ambas metodologías analíticas.

\section{Generalidades arqueológicas}

Los materiales arqueológicos analizados proceden de dos sitios estratificados (Cerro Casa de Piedra 7 y Cueva del Mylodón Norte 1) y uno de superficie (Playa Cisnes 2) situados en el NO de la provincia de Santa Cruz (Figura 1).

El estudio tipológico del conjunto artefactual de los sitios permitió observar que acompañando a las puntas triangulares apedunculadas (Figura 2 a-j), en cierto número de instrumentos unifaciales del total del conjunto lítico, se repetía un llamativo rasgo tecnológico (Civalero 2014, 2016, Sacchi et al. 2016, Nami y Civalero 2017). Tal como se ilustra en la figura 2, se caracteriza por tener lascados unifaciales, parcial o totalmente extendidos, con el objeto de formatizar una de las caras del instrumento (Figura 2 k-m, r-t). Sin descartar otra clase de utensilios, este estilo de producción era principalmente utilizado para confeccionar raspadores y raederas de materias primas variadas (Civalero 2016). Con la meta de explorar su secuencia de producción, en una primera etapa se efectuó un estudio experimental el cual permitió considerar que, en efecto, se trataba de un particular

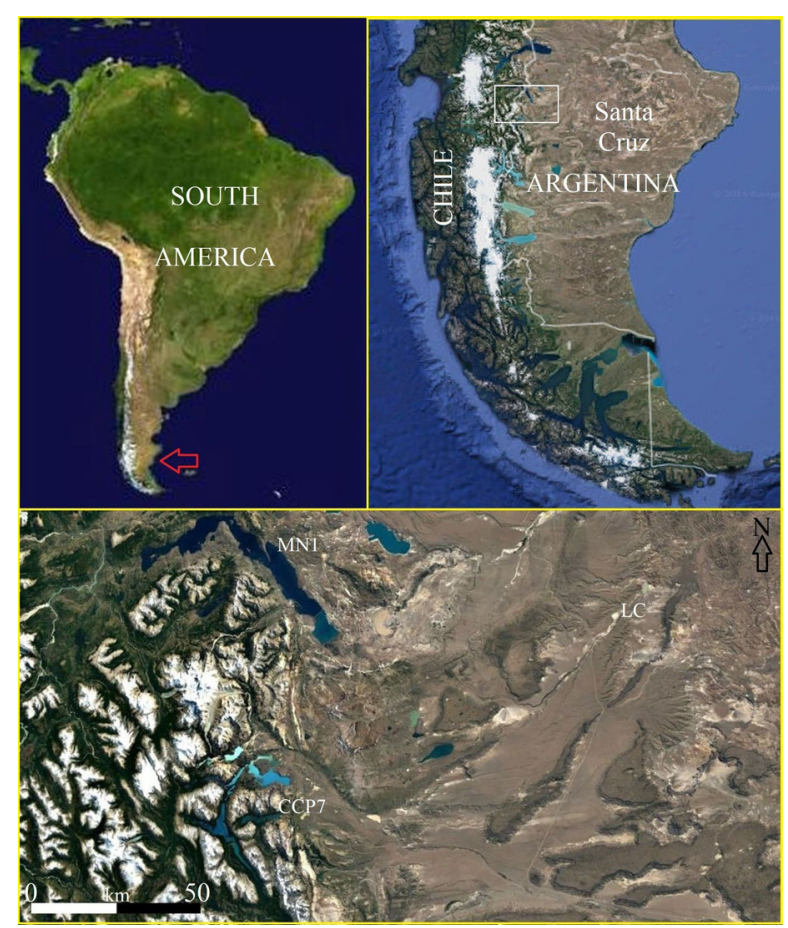

Figura 1. Ubicación del área. Sitios Laguna Cisnes (LC); Cueva Milodón Norte 1 (CMN1); Cerro Casa de Piedra 7 (CCP7).

Figure 1. Location of the area. Sites Laguna Cisnes (LC); Cueva Milodón Norte 1 (CMN1); Cerro Casa de Piedra 7 (CCP7). patrón tecnológico de reducción (Nami y Civalero 2016, 2017). De esa manera, uno de sus rasgos más notables es que la forma-base, mayoritariamente la cara dorsal de una lasca, se talla con el objetivo de conformar una superficie aproximadamente convexa y un espesor relativamente uniforme (Civalero 2016). Vale la pena mencionar que en otros sitios de la Patagonia meridional también se identificaron utensilios confeccionados de una manera semejante (Figura $2 \mathrm{n}-\mathrm{q}$ ).

Las investigaciones previas permitieron hipotetizar que: 1) los lascados profundos corresponden a una acción preconcebida, planeada y deliberada que forma parte del proceso de conformación del producto final (Nami y Civalero 2016, 2017); 2) no son accidentales ni resultan de las reactivaciones, acción a la cual normalmente eran sometidos esta clase de implementos (Dibble 1995, Baena Preysler y Carrión Santa Fe 2010); 3) esta manera de confeccionar instrumentos unifaciales correspondería a un estilo técnico particular de las ocupaciones del Holoceno temprano (Civalero 2016, Nami y Civalero 2016, 2017), al menos en una porción de la Patagonia centromeridional y del noroeste de Santa Cruz en particular.

\section{Metodología}

Con el propósito de ampliar, controlar, y contrastar las hipótesis planteadas se emplearon dos metodologías de estudios líticos diferentes que brevemente se detallarán a continuación.

\section{Experimentación}

La experimentación se utiliza con el propósito de evaluar y explorar las peculiaridades tecno-morfológicas consideradas inicialmente (Nami y Civalero 2017) y generar información básica para tener una aproximación más profunda del análisis diacrítico en los especímenes arqueológicos.

Las lascas utilizadas como soportes se extrajeron de cuatro nódulos expresamente reducidos para este experimento: 1) de basalto, procedente de Antofalla (Catamarca); 2-3) de pórfiro riolítico del Departamento Magallanes, Santa Cruz; 4) de vidrio caramelo de origen industrial y algunas lascas de vidrio tipo Pyrex, ambos hechos en Buenos Aires. Estos materiales poseen propiedades de laboreo semejantes a las de los originales, aunque con algunas diferencias cualitativas. De hecho, en una escala empírica cuyos valores tienen un rango de 0,5 a 5.5 y de 1,0 a 5,0 para la mayoría de las rocas (Callahan1979: 16), las dos primeras tienen una gradación de 4,5-5 y 4,0-4,5 respectivamente, mientras que los vidrios de 1,0 y 1,5-2. La figura 3 ilustra algunos de los nódulos y lascas obtenidas.

La técnica de talla empleada fue percusión directa con diferentes variantes, tanto en su aplicación como en la forma de sostén del artefacto trabajado. Tal como se 


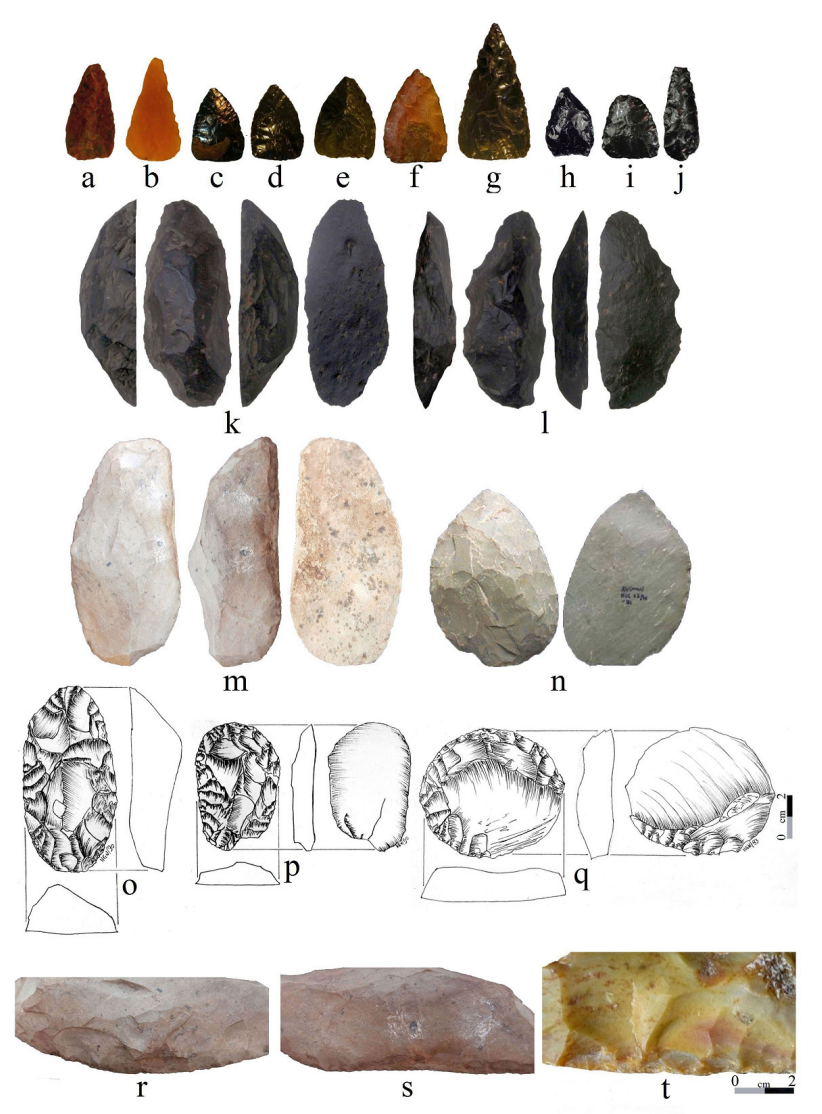

Figura 2. Ejemplos de instrumentos arqueológicos. a-j) puntas de proyectil triangulares apedunculadas procedentes de CCP7 capas 12 (a-e), 14 (f), 18 (g), PC2 (h-j). k-q) Instrumentos unifaciales. j-k) de las capas 14 y 18 de (CP7; m) CMN1; n) Alero del Valle; o-q) Cueva del Medio; r-t) Detalle de algunos de los instrumentos unifaciales analizados.

Figure 2. Examples of archaeological artefacts: a-i) triangular projectil points from CCP7 site level 12 (a-e), 14 (f), 18 (g), PC2 site $(h-j) . k-q)$ unifacial artefacts. $j$-k) from levels 14 and 18 CCP7 site; ( $m$ ) from CMN1 site; ( $n$ ) from Alero del Valle site; (o-q) from Cueva del Medio site; $(r-t)$ some details of analyzed unifacial tools.

observa en la figura 4, las variedades fueron las siguientes: a) con la mano libre, b) con la muñeca y el dorso de la mano apoyando sobre el muslo y los dedos colocados en la cara trabajada, c) afirmando la pieza trabajada en el muslo. La aplicación de esta técnica se efectuó con percutores orgánicos blandos de asta, como así también blandos, semi-duros y duros de diferentes rocas y pesos (Nami 2002). Durante la tarea se emplearon dos protectores, uno de cuero y el otro de neopreno.

En la descripción de la secuencia de producción de los artefactos experimentales se consideró útil la cadena de pasos generales propuesta más de tres décadas atrás a saber: Extracción, formatización y regularización (Nami 1984). En la de extracción se obtiene la forma-base a partir de núcleos o simplemente recogiendo guijarros, clastos, o cualquier otro elemento natural adecuados

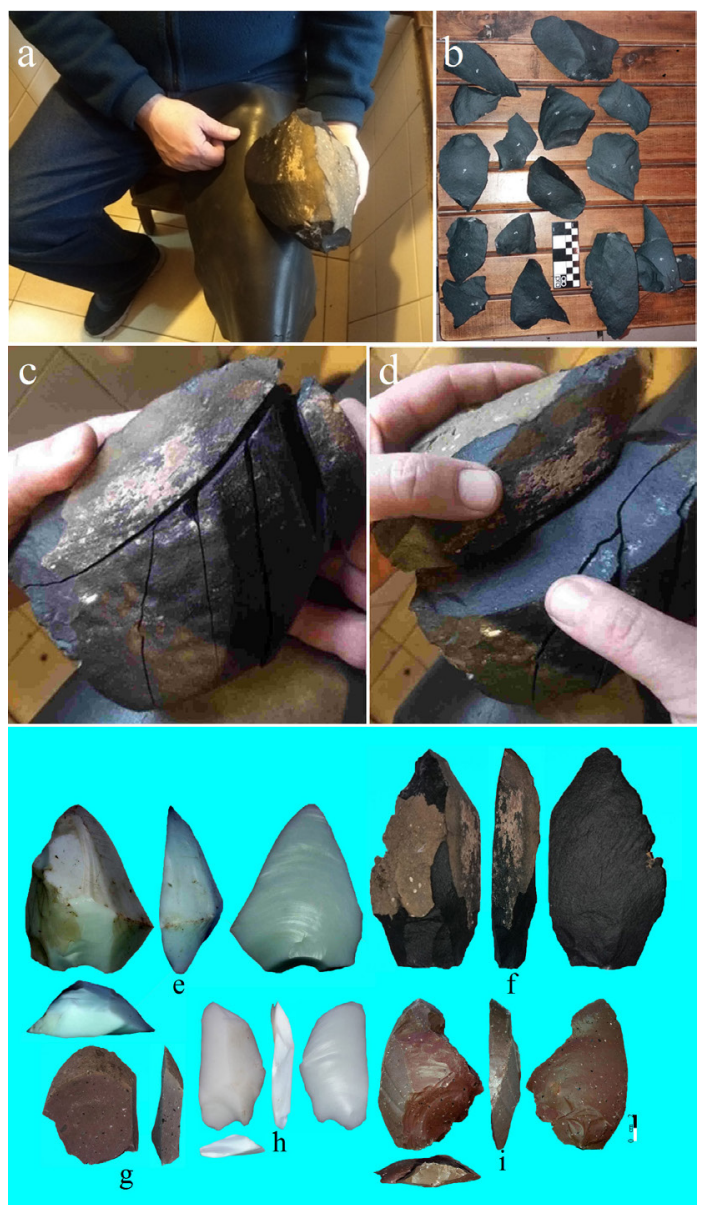

Figura 3. Ejemplos de la etapa inicial en la manufactura experimental de los artefactos bajo estudio. Nódulo de basalto procedente de Antofalla (a), totalidad de las lascas extraídas (b) y ensambladas (c-d), e-i) algunas de las formas-bases empleadas.

Figure 3. Examples of the initial step of experimental manufacture. Basalt nodule from Antofalla (a); the totality of the detached b) and fitted flakes (c-(d); e-i) blanks used.

para la conformación ulterior del instrumento. En la formatización el artefacto es tallado utilizando distintas técnicas con el objeto de ir configurando el producto terminado. Tecnológicamente, según la técnica de reducción (sensu Flenniken 1984) puede ser algún estadio de manufactura temprano o medio. Finalmente, mediante la regularización el instrumento adquiere la forma final aplicando una talla más fina y delicada. Estas etapas no son obligatorias, ya que puede haber muchos casos de extracción seguido de regularización.

Debido a la naturaleza de los artefactos arqueológicos, las lascas experimentales fueron extraídas azarosamente utilizando plataformas naturales, y/o las superficies resultantes de extracciones previas o con un mínimo de preparación. La talla se realizó sentado en una silla, los nódulos y núcleos fueron envueltos con el protector y la percusión fue aplicada apoyándolo en el muslo de la pierna izquierda o en su parte externa. Los percutores 


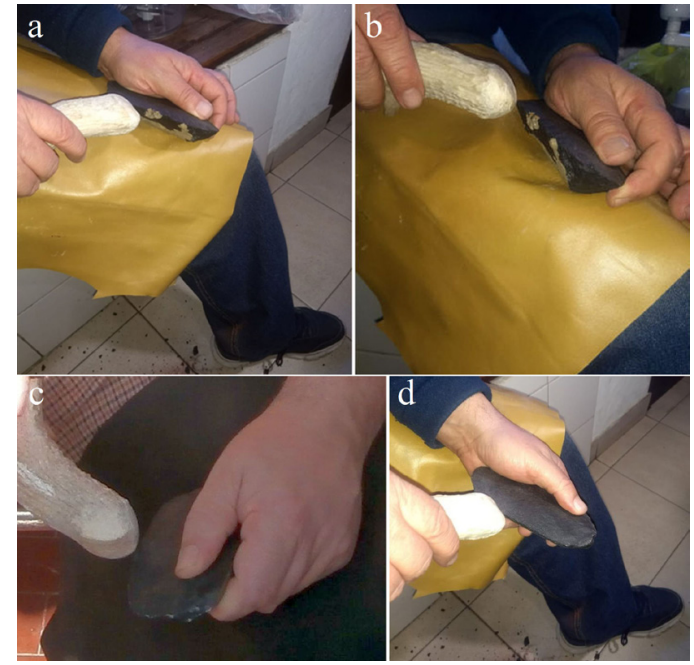

Figura 4. Variantes de percusión directa aplicada con un asta de ciervo y de las formas de sostén apoyando la pieza y sosteniendo la muñeca sobre el muslo del mismo lado. Ambas fueron respectivamente empleadas durante las etapas de formatización (a-b) y regularización (c-d).

Figure 4. Variants of direct percussion applied with an antler billet and the holding positions supporting the piece and holding the wrist on the thigh on the same side. Both were respectively used during the shaping step $(a-b)$ and final shaping $(c-d)$.

utilizados sobre el basalto y el pórfiro fueron de rocas duras cuyo peso era de 400 y $500 \mathrm{~g}$. Aunque en algunos casos, dado que al principio dificultó la extracción por su resistencia a la fractura, un peso $\geq 800 \mathrm{~g}$ hubiera sido recomendable. En el caso de los vidrios se empleó un percutor de roca blanda de $800 \mathrm{~g}$. Los ángulos de plataforma adecuados para la extracción variaron entre $\sim 80^{\circ}$ y $85^{\circ}$; valores mayores dificultaron la tarea, especialmente en el material catamarqueño.

Las lascas obtenidas del nódulo de basalto de Antofalla no formaron conos de fuerza pronunciados como sería de esperar en rocas más frágiles, sino que fueron planos y difusos, tal como ocurre en otros materiales con propiedades diferentes a las óptimas para confeccionar instrumentos líticos (Nami 2015). Tampoco se conformaron ondas de aplicación de la fuerza marcadas ni estrías. Se produjeron muchas lascas adventicias, algunas de las cuales quedaron en el negativo de la extracción. El pórfiro mostró cualidades diferentes, uno se comportó de manera parecida al basalto mientras que el otro generó atributos semejantes a los observados en los materiales óptimos, situación ya esperada con los vidrios.

La mayoría de la totalidad $(n=15)$ de lascas obtenidas del nódulo de basalto (Figura 3b) tenían características adecuadas para confeccionar instrumentos unifaciales aplicando distintas estrategias de talla. No obstante, se seleccionaron unas pocas consideradas útiles con la meta de reproducir los artefactos bajo estudio (e.g. Figura 3f).
De los núcleos de pórfiro y vidrio caramelo se obtuvieron 3, 6 y 7 lascas respectivamente, no todas aptas para este experimento, razón por la cual solamente se utilizaron algunas (e.g. Figura 5g).

Las piezas experimentales se confeccionaron de la manera descripta anteriormente (Nami y Civalero 2017). Una vez extraídos y seleccionados los soportes, la formatización se efectuó aplicando distintas variantes de percusión directa y formas de sostén. Con el objetivo de eliminar las irregularidades y filos naturales de la forma-base mayormente se empleó la variante de mano libre o apoyándola en el muslo. Luego, la talla unifacial extendida que cubrió con lascados profundos la cara dorsal de manera parcial o total se efectuó con el percutor de asta. A veces, se realiza una acción similar sobre la cara ventral para emparejarla y eliminar abultamientos, especialmente los del cono de percusión (Nami y Civalero 2017: Figura4a). En esta tarea, las plataformas fueron escasamente preparadas abradiendo levemente el filo. Si bien los desechos de talla tienen formas variadas, es importante mencionar que algunos son semejantes a los que resultan del trabajo bifacial (Figura5 f-h).

Finalmente, una vez ya conformada la forma-base, se regulariza en algún instrumento, ya sea raspador o raedera en general. La regularización se efectuó con percusión directa aplicada con percutores blandos de asta o piedra. También se empleó la variedad de percusión por contragolpe (de Mortillet 1910), la cual consiste en apoyar el borde a retocar sobre una piedra y aplicar golpes con un percutor en la cara dorsal del filo regularizado. De acuerdo a las características de la superficie de la cara dorsal, la secuencia de remoción de lascas fue relativamente continua o azarosa (Figura5).

Además, con el propósito de indagar si los lascados extendidos pueden resultar de la percusión comúnmente utilizada para retocar el filo de las raederas, un borde de una de las lascas de vidrio blanco (Figura 3e) fue tallada empleando un percutor de piedra blanda de material calcáreo (Nami 2015: Figura 3d) con percusión apoyando la muñeca sobre el muslo. Luego, y con la meta de comparar la forma de los lascados, se trabajó el borde opuesto con la misma forma que el resto de las piezas experimentales. De esta manera, adyacente a la porción más gruesa y abrupta de la lasca se la apoyó en el muslo y aplicó la técnica con un percutor semi-duro de piedra de roca cuarcítica.

\section{Análisis diacrítico.}

El estudio consiste en el reconocimiento de la secuencia de extracción o remoción de lascas para comprender los rasgos implícitos en su superposición. Así, se efectúa una lectura diacrítica que pretende generar un saber fiable concerniente a las series o esquemas de confección y por lo tanto permite conocer gran parte del proceso tecnológico (Baena Preysler y Cuartero 2006). El análisis de los negativos de lascado constituye uno de los objetivos 
básicos de la metodología, pues de este modo es posible establecer individualmente la anterioridad o posterioridad de las extracciones. Asimismo, permite reconocer la disposición de cada secuencia de remoción dentro de una serie en la que no existen cambios significativos en los procedimientos de talla y en el cual la concepción (sensu Pelegrin, en Callahan 1982) del artesano se mantiene con la misma finalidad.

El reconocimiento macroscópico de la secuencia tecnológica se llevó a cabo utilizando algunos de los criterios propuestos por Baena Preysler y Cuartero (2006). El punto de partida del análisis diacrítico está dado por la observación de las ondas y estrías que se manifiestan luego de la extracción de cada lasca. Las ondas comienzan en el lugar de impacto del percutor a partir del cual se expanden y amplían cuando se distancian del mismo; asimismo, a medida que se alejan de ese punto, las estrías se irradian hacia los extremos. Según los investigadores citados, el orden de cada secuencia se puede determinar mediante la observación de la ruptura o cambio abrupto en la simetría de forma en la superficie del lascado. Asimismo,

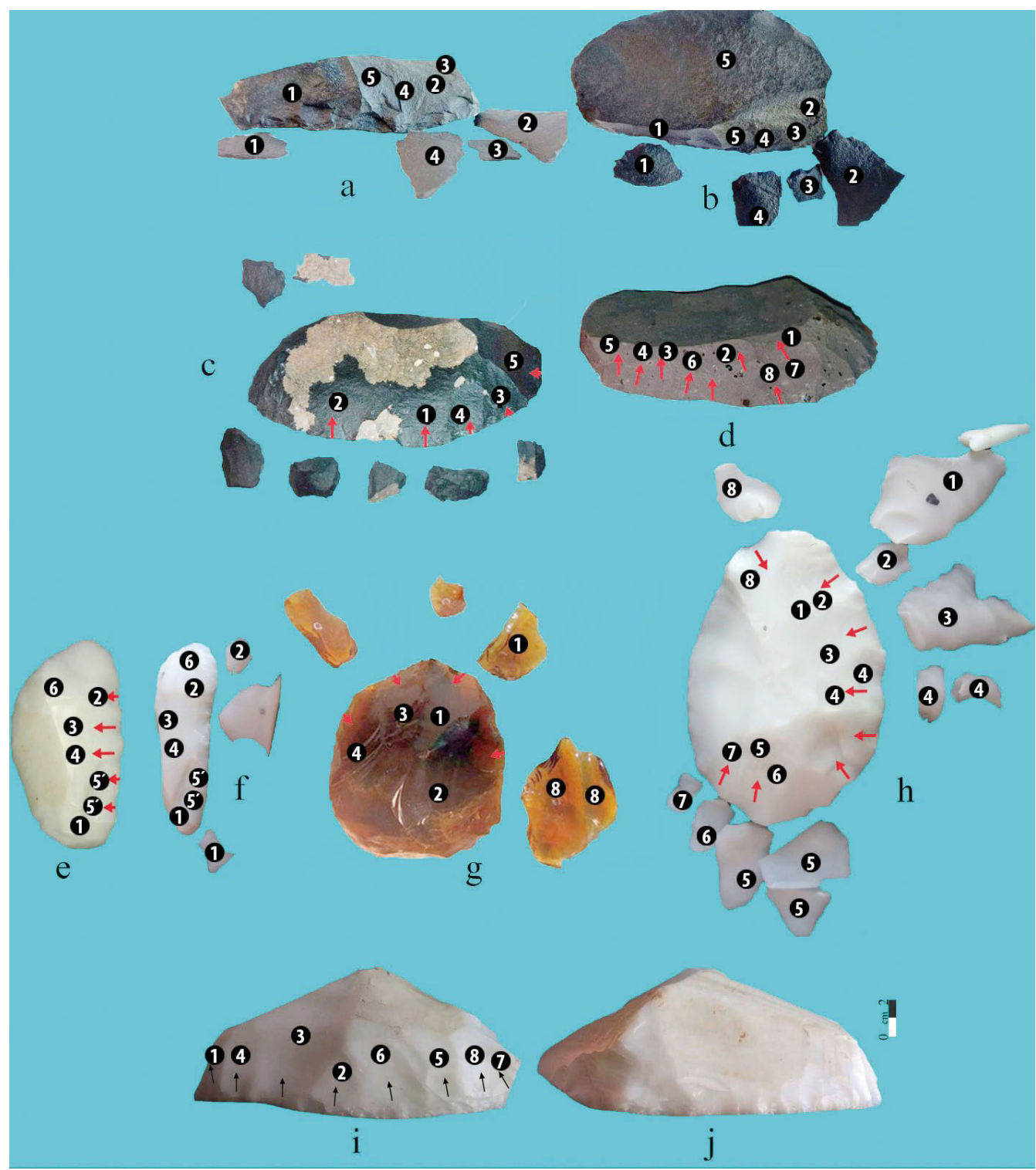

Figura 5. Ejemplos de piezas experimentales. a-h) secuencia de remoción de lascas con sus respectivos desechos registrados durante la formatización de la forma-base, a-b) muestra dos vista de la misma pieza, e-f) ilustra la misma pieza formatizada y regularizada, i-j) Ambos bordes de la misma pieza experimental mostrando el análisis diacrítico del confeccionado con formatización previa a la regularización (i) comparado con el opuesto en el que falta ese procedimiento (j).

Figure 5. Some examples of experimental artefacts. $a$-h) detached flakes with their debris during the reduction sequence, $a-b)$ both sides of the artefact, e-f) the same artefact by detaching covering flakes and then finished by retouches, $i-j)$ both sides of the same piece showing the diacritic analysis on the edge with previous shaping (i), and the other side lacking this procedure (j). 
la simetría de las ondas de aplicación de la fuerza da pistas para este análisis, pues se ven interrumpidas por la nueva cuando se observa minuciosamente la yuxtaposición de las extracciones (Baena Preysler y Cuartero 2006). Otro atributo importante a tener en cuenta es aquello que los autores mencionados llaman "rebaba" para referirse a la micro-topografía existente en las aristas o nervaduras de los negativos. Muy sensible al tacto, la misma se manifiesta en la porción latero-distal de la remoción más reciente que se sobrepone a las anteriores. Entonces, a partir de las ondas de aplicación de la fuerza, la forma de las estrías y la rebaba latero-distal se establecería la superposición de las secuencias de lascados. De este modo, las estrías del borde en general son mucho más notables que las existentes en el centro, y la superposición se identifica a partir de la verificación táctil de sus aristas. Así, desde el negativo más reciente se encontraría una mayor resistencia al tacto, notándose más suave hacia el anterior.

Los análisis mencionados no se podrían llevar a cabo sin la reproducción experimental, pues la replicación controlada de las piezas arqueológicas representa una de las mejores herramientas para comprender el proceso de lectura tecnológico (Baena Preyler y Cuartero 2006). En otras palabras, es un paso previo e insoslayable para organizar hipótesis que luego serán contrastadas con el material arqueológico. De esta manera, las posibilidades surgidas de la aplicación del método experimental se controlaron con la utilización de esquemas diacríticos de análisis lítico aplicado tanto a materiales actuales como a los arqueológicos. A partir de esta metodología exploraremos tanto técnicas de talla como así también secuencia/s y/o esquema/s de confección de los artefactos estudiados.

Puesto que el principal interés de la investigación fue averiguar y profundizar la conformación previa a la regularización, con la meta de monitorear con demasía de detalles y tener una idea acabada de la formatización, durante el experimento se registró el orden de la secuencia de remoción de lascas (e.g. Figura 5). De esta manera, a medida que se removía cada una se la enumeró con el objeto de tener un control minucioso de las series de extracciones (5a-h). Asimismo, se efectuó un examen diacrítico en varias piezas trabajadas en sesiones experimentales anteriores (Figura 5 i). Por ende, se generaron datos de primera mano e información básica vinculada con la conformación previa a la regularización, lo cual redundó en el estudio diacrítico de los especímenes arqueológicos. De hecho, una vez confeccionado el material de referencia actualístico-experimental se llevó a cabo un escrutinio similar en los vestigios del pasado. Algunos ejemplos de las piezas experimentales y arqueológicas analizadas con este método se ilustran en las figuras 5 y 6 . Las flechas en lascados señalan la dirección en la que fue desprendida cada lasca en la formatización; los números indican el orden de las extracciones conformando una serie y las

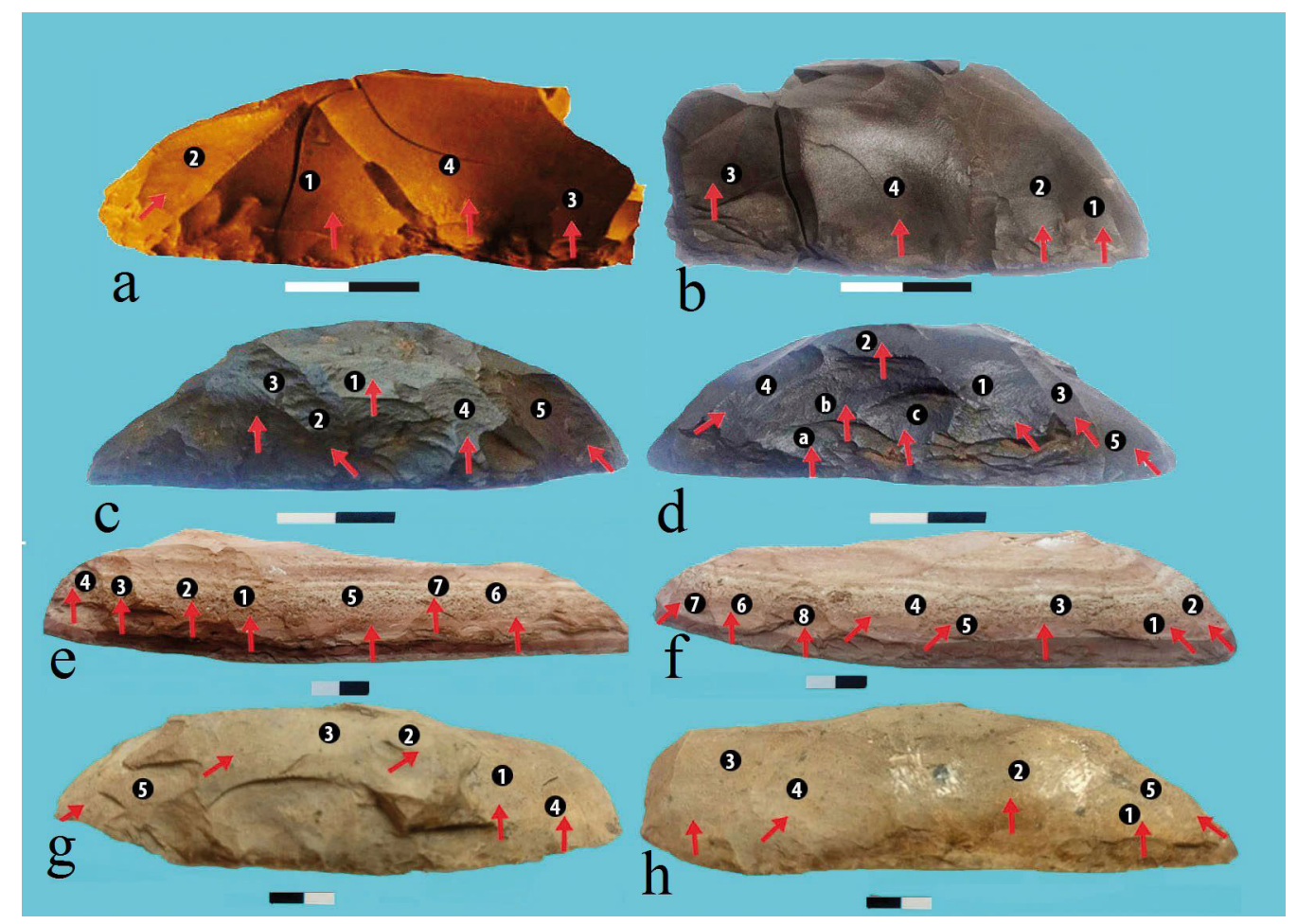

Figura 6. Ejemplos de análisis diacríticos efectuados sobre los filos laterales de raederas arqueológicas. a-b) CCP7, c-d) CMN1. Las escalas representan $2 \mathrm{~cm}$.

Figure 6. Examples of diacritic analysis carry out on the lateral edges of archaeological side scrapers. a-b) CCP7 site, c-d) CMN1 site. The scales represent $2 \mathrm{~cm}$. 
letras en una misma pieza representan otra serie dentro de un mismo instrumento.

\section{Resultados}

La experimentación y el análisis diacrítico arrojaron luz sobre la secuencia de reducción de los utensilios bajo estudio; además con aspectos vinculados a las posibles variantes técnicas, formas de sostén, e implementos de talla. Desde el primer punto de vista se confirma que, en efecto, una vez obtenida la forma-base aplicando percusión directa, luego de emparejar el borde con percusión con la mano libre, se la formatizaba tallando mayormente su cara con la misma técnica, pero con variantes para lograr lascados parcial o totalmente extendidos; una vez logrado ese cometido, se finalizaba el instrumento utilizando la misma técnica, pero variando la forma de sostén de la pieza trabajada. En este sentido, de acuerdo a los materiales se podría haber utilizado percusión directa aplicada con percutores de piedra blandos o semi-blandos y blandos de material orgánico, particularmente de tejido óseo. Vale la pena mencionar que se observaron diferencias de forma en los negativos de lascado resultantes de las dos variantes empleada en la manufactura de los filos de la pieza confeccionada en Pyrex blanco. Cuando se empleó percusión con mano libre o afirmando la muñeca y la mano sobre el muslo, si bien se obtuvieron algunos lascados profundos, en general fueron más cortos, escamosos e irregulares (Figura 5 j), mientras que los obtenidos apoyando la pieza en el muslo fueron más penetrantes en el espesor del soporte y las ondas de aplicación de la fuerza menos marcadas y con aristas relativamente paralelas. La forma también fue diferente, vale decir más regulares, cubrientes y profundos (Figura 5 i).

El estudio diacrítico de esta pieza mostró que durante la etapa de formatización, la serie de extracción no fue continua como se aprecia en la figura 5i. Este análisis en las reproducciones experimentales permitió registrar que en la formatización las series empleadas conformaron artefactos espesos con aristas sinuosas e irregulares, luego emparejadas y eliminadas en la regularización. En los artefactos arqueológicos el estudio diacrítico mostró que, en esta etapa, la talla fue aplicada en series sucesivas y convergentes unidireccionales sobre la cara dorsal. En algunos casos se observa una superposición de negativos de lascado evidenciando el empleo de dos o más series de extracciones aplicadas sin continuidad (Figura 6). Además, mostró homogeneidad técnica, evidenciada por un patrón de talla similar que sugiere cierta estandarización técnico-morfológica. Las formas-base utilizadas en el material arqueológico examinado son lascas espesas probablemente seleccionadas ex profeso.

\section{Discusión y conclusión}

En resumen, tal como era de esperar las metodologías utilizadas se complementaron adecuadamente para profundizar en el conocimiento de la secuencia de reducción de los artefactos bajo estudio. En este aspecto, la reproducción experimental de los instrumentos arqueológicos posibilitó una aproximación detallada, proporcionando nuevos datos, especialmente durante la etapa de formatización. De esta manera generó información básica para conocer las etapas necesarias en su manufactura e hipotetizar sobre las variantes de aplicación de percusión, formas de sostén e implementos de talla.

El análisis diacrítico de los especímenes experimentales permitió observar y registrar, con claridad, la secuencia de remoción de lascas en el proceso. De este modo se constató que, con anterioridad a la regularización del instrumento, se presentaban una, dos o más series de extracciones profundas, cuyo objetivo no estaba relacionado con la conformación del filo, sino con la preparación de una de las caras, precisamente la dorsal del soporte empleado. Esta metodología aplicada al estudio de los vestigios arqueológicos, proporcionó resultados semejantes. Esta clase de formatización está presente en todos los especímenes estudiados. Por ejemplo, la figura 6 filustra que un borde de la pieza analizada tiene una serie de 8 extracciones dispuestas en una secuencia relativa que podría seguir una dirección de derecha a izquierda, mientras que el opuesto tiene una serie de 7 extracciones sin un patrón que las defina (Figura 6 e). No obstante, está claro que ambas se efectuaron durante la formatización previamente a la regularización.

Lo anterior robustece las hipótesis planteadas y corrobora algunas otras. En este último caso, se mostró que los lascados total o parcialmente extendidos corresponden a una acción preconcebida y deliberada tendiente a la formatización del producto final y no son accidentales ni resultan de las reactivaciones. Así, se refuerza la posición de que este modo de confeccionar utensilios correspondería a un estilo técnico compartido por las ocupaciones del Holoceno temprano en la Patagonia centro-meridional en general y del noroeste de Santa cruz en particular.

Además de reportar anteriormente el descubrimiento de esta peculiar técnica de manufactura en la región, se informó el posible empleo de procedimientos parecidos en otros lugares del cono sur y de Sudamérica (Nami y Civalero 2017). Es significativo apuntar que poco después de la presentación de esta pesquisa en el simposio de arqueología experimental reportado en este volumen, se efectuaron investigaciones similares en conjuntos instrumentales de la misma edad recolectados en sitios del Macizo del Deseado en la provincia de Santa Cruz (Hermo et al. 2018). Dichos análisis concluyeron que, en efecto, esta peculiar secuencia de reducción, también fue empleada en dicha localidad. Asimismo, estudios diacríticos efectuados sobre un conjunto de raederas 
datadas en el último milenio del Pleistoceno en el estado de Goiás en centro-oeste de Brasil (Viana Sibeli y Torres Borges 2014) permitieron demostrar que también fue utilizada una manera de confección semejante a la de Patagonia centro-meridional.

Buenos Aires, 28 de marzo de 2019

\section{Agradecimientos}

Estamos profundamente agradecidos a las siguientes personas e instituciones: CONICET, Universidad de Buenos Aires e Instituto Nacional de Antropología y Pensamiento Latinoamericano por apoyar nuestras investigaciones arqueológicas y experimentales en la Patagonia; Parques Nacionales y a los guías de Cueva de las Manos; la Agencia Nacional de Promoción Científica y Tecnológica (PICT 06-2488 dirigido por C. Aschero) por financiar los trabajos de campo; todos los compañeros que compartieron campañas arqueológicas en laguna Cisnes, CCP7 y CMN1; la familia Otamendide Estancia El Bagual por las gentilezas otorgadas al equipo y por permitirnos las excavaciones en otra de sus estancias. Lic. Carolina Avila y su equipode la Asociación Identidad de la localidad de Perito Moreno por la presencia permanente de dicha Asociación colaborando con las necesidades del equipo. A Mercedes Cuadrado por la lectura crítica del manuscrito.

\section{Referencias}

Baena Preysler, J. y Cuartero, F. (2006). Más allá de la tipología lítica: Lectura diacrítica y experimentación como claves para la reconstrucción del proceso tecnológico.José Manuel Maillo y Enrique Baquedano (Eds.) Miscelánea en homenaje a Victoria Cabrera. Zona Arqueológica 7(1): 144-161.

Baena Preysler, J. y Carrión Santafé, E. (2010). Experimental Approach to the Function and Technology of Quina Side-Scrapers. En H. G. Nami (Ed.), Experiment and Interpretation of Traditional Technologies: Essays in Honor of Errett Callahan (pp. 171-202). Buenos Aires: Ediciones de ArqueologíaContemporánea.

Callahan, E. (1979). The Basics of Biface Knapping in the Eastern Fluted Point Tradition. A Manual for Flintknappers and Lithic Analysts.Archaeology of Eastern North America, 7: 1-180.

Callahan, E. (1982). An Interview with Flintknapper Jacques Pelegrin. Contract Abstracts 3(1): 62-70.

Civalero, M. T. (2016). Propuesta metodológica para el análisis del material lítico del sitio Playa Cisnes 2, provincia de Santa Cruz, Patagonia argentina. En F. Mena (Ed.) Arqueología de la Patagonia: de mar a mar (pp. 235-244). Coyhaique: Ediciones CIEP/Ñire Negro Ediciones.

deMortillet, A. (1910). Le Travail de la Pierre auxtempspréhistoriques (Premièrepartie). Revue de l'Ecoled'Anthropologie de Paris, 20, 1-21.

Dibble, H. L. (1995). Middle Paleolithic Scraper Reduction: Background, Clarification, and Review of the Evidence to Date. Journal of Archaeological Method and Theory, 2: 299-368.
Flenniken, J. J. (1984). The Past, Present, and Future of Flintknapping: An Anthropological Perspective.Annual Review of Anthropology, 13(1), 187-203.

Hermo D., Mosquera, B., Vargas Gariglio, J. y Pérez, A. (2018). Indicadores tecnológicos de colonización en contextos de superficie del Macizo del Deseado (Santa Cruz, Argentina). Libro de Resúmenes del 9 Simposio Internacional el Hombre Temprano en América. La gente y sus lugares (pp. 66-67). Necochea.

Nami, H.G. (1984). La tecnología lítica y una nueva propuesta nomenclatoria. Arqueología Contemporánea I (2): 21-25.

Nami, H. G. (2002). Comentario al libro "Tecnología Lítica Experimental. Introducción a la Talla de Utillaje Prehistórico" por J. Baena Preysler, BAR International Series 721, Oxford, 1998. Trabajos de Prehistoria 59 (1): 182-185, Madrid.

Nami, H. G. (2010). Theoretical Reflectionson Experimental Archaeology and Lithic Technology. En Nami, H. G. (Ed.) Experiments and Interpretation of Traditional Technologies: Essays in Honor of Errett Callahan (pp.91-168). Ediciones de Arqueología Contemporánea. Buenos Aires.

Nami, H. G. (2011). Fundamentos teóricos y epistemológicos sobre arqueología y tecnología lítica experimental. Arqueología Rosarina Hoy 3(1): 75-98.

Nami, H. G. (2015). Experimental Observations on some Non-Optimal Materials from Southern South America. Lithic Technology, 40: 128-146.

Nami, H. G. (2018). Theoretical and Epistemological Thoughts on Archaeology and Experimental Lithic Technology. Journal of Research in Philosophy and History 1(2): 139-165.

Nami, H. G. y Civalero, M. T. (2016). Experimentos para explorar la manufactura de instrumentos unifaciales peculiares del noroeste de la provincia de Santa Cruz. Libro de Actas del XIX Congreso Nacional de Arqueología Argentina. Serie Monográfica y Didáctica, 54: 769-772. San Miguel de Tucumán.

Nami, H. G. y Civalero, M. T. (2017). Distinctive Unifacial Technology during the Early Holocene in Southern South America. Archaeological Discovery 5: 101-115.

Sacchi M., Bozzuto, D., Horta, L.,Fernández, N., De Nigris, M.,Civalero, M.T., Aschero, C. (2016). Dataciones y circulación humana: influencia de las fluctuaciones del sistema lacustre Pueyrredón-Posadas durante el Holoceno. Andes (Salta) (27) 27. CEPIHA (Centro Promocional de Investigaciones en Historia y Antropología).

Viana Sibeli, A. y Borges, C. T. (2014). Compreendendo ferramentas líticas a partir das persistências e das variabilidades técnicas. Estudo de caso dos sítios GO-CP-17 e MT-SL-31, Região Centro Oeste do Brasil. En (Farías, M. \&Lourdeau, A. Eds.) Peuplement etModalitésd'Occupation de l'Amérique du Sud: l'Apport de la Technologie Lithique (pp.65-95). Prigonrieux: @rchéo-éditions.com and Impr. Copy-média. 